Sección Control / Control

Artículos de investigación / Research paper

\title{
Macromorfología y crecimiento radial de cepas de hongos entomopatógenos suplementado con polvo de lepidópteros
}

\author{
Macromorphology and radial growth of entomopathogenic fungi strains \\ suplement with lepidoteran powder
}

\author{
CIPRIANO GARCÍA-GUTIÉRREZ1; MARINA ISABEL GARCÍA-GUAJARDO²; \\ GUADALUPE VEJAR-COTA ${ }^{3}$; LORENZO MEZA-GARCÍA ${ }^{4}$; \\ JESÚS ALICIA CHÁVEZ-MEDINA ${ }^{5}$
}

\begin{abstract}
${ }^{1}$ Doctor, Instituto Politécnico Nacional, COFAA-CIIDIR IPN Unidad Sinaloa, Boulevard Juan de Dios Batíz Paredes 250, C. P. 81101, Col. San Juachin, Guasave, Sinaloa, México, garciaciprian@hotmail.com, https://orcid.org/0000-0002-8967-3679. ${ }^{2}$ Bióloga, Universidad Autónoma de Occidente Región Guasave, Sinaloa, México, marina.guajardo121@gmail.com, https://orcid.org/0000-0002-0750-7651. ${ }^{3}$ Doctor, Instituto Politécnico Nacional, CIIDIR IPN Unidad Sinaloa, Boulevard Juan de Dios Batíz Paredes 250, C. P. 81101, Col. San Juachin, Guasave, Sinaloa, México, vejargpe@hotmail.com, https://orcid. org/0000-0002-1673-2009. ${ }^{4}$ Doctor, Universidad Autónoma de Occidente, Guasave, Sinaloa, México, lorenzo meza@prodigy.net.mx, https://orcid.org/00000002-7656-561X. ${ }^{5}$ Maestra, Instituto Politécnico Nacional, CIIDIR IPN Unidad Sinaloa, Boulevard Juan de Dios Batíz Paredes 250, C. P. 81101, Col. San Juachin, Guasave, Sinaloa, México, alchavez@ipn.mx, https://orcid.org/0000-0002-8552-2590.
\end{abstract}

Autor para correspondencia: Cipriano GarcíaGutiérrez, Doctor, Instituto Politécnico Nacional, COFAA-CIIDIR IPN Unidad Sinaloa, Boulevard Juan de Dios Batíz Paredes 250, C. P. 81101, Col. San Juachin, Guasave, Sinaloa, México,garciaciprian@hotmail.com, https:// orcid.org/0000-0002-8967-3679.

Citación sugerida / Suggested citation: GARCÍA-GUTIÉRREZ, C.; GARCÍAGUAJARDO, M. I.; VEJAR-COTA, G.; MEZAGARCÍA, L.; CHÁVEZ-MEDINA, J. A. 2020. Macromorfología y crecimiento radial de cepas de hongos entomopatógenos suplementado con polvo de lepidópteros. Revista Colombiana de Entomología 46 (1): e10164. https://doi. org/10.25100/socolen.v46i1.10164

Recibido: 17-sep-2018

Aceptado: 8-jun-2019

Publicado: 5-ago-2020

Revista Colombiana de Entomología

ISSN (Impreso): 0120-0488

ISSN (En línea): 2665-4385

https://revistacolombianaentomologia.univalle.edu.co/

Open access

Publicadores / Publishers:

Sociedad Colombiana de Entomología SOCOLEN (Bogotá, D. C., Colombia) http://www.socolen.org.co Universidad del Valle (Cali, Colombia) http://www.univalle.edu.co/

(C) 2020 Sociedad Colombiana de Entomología - SOCOLEN y Universidad del Valle - Univalle
Resumen: Se estudió la macromorfología de cepas de Beauveria bassiana (B1), Metarhizium anisopliae (M1) e Isaria javanica (HPI-210) y se determinó su crecimiento radial a temperaturas de $25,28,30,35$ y $40{ }^{\circ} \mathrm{C}$ en medio PDA suplementado con polvos de Diatraea considerata, Spodoptera frugiperda y Galleria mellonella. Los hongos se inocularon adicionando $2 \mu \mathrm{lde}$ una suspensión con $1 \times 10^{7}$ esporas/ml. Después de 15 días se midió el crecimiento radial de las cepas en $\mathrm{mm} / \mathrm{d}$. Para determinar la interacción entre las tres cepas con el polvo de los tres insectos más el control PDA y las cinco temperaturas, se usó un análisis factorial $3 \times 4 \times 5$; los datos del crecimiento de los hongos fueron analizados mediante un ANOVA y una prueba de Tukey. Se encontraron diferencias estadísticas significativas en el crecimiento a diferente temperatura, respecto al control; el crecimiento óptimo en los tres hongos fue a $28{ }^{\circ} \mathrm{C}$, la cepa B1 suplementada con polvo de $D$. considerata creció $2,76 \pm 0,06 \mathrm{~mm} / \mathrm{d}, \mathrm{M} 1$ con $G$. mellonella $2,77 \pm 0,02 \mathrm{~mm} / \mathrm{d}$, y HPI-210 con D. considerata $3,27 \pm 0,06 \mathrm{~mm} / \mathrm{d}$. La cepa B1 creció $0,45 \pm$ $0,03 \mathrm{~mm} / \mathrm{d}, \mathrm{M} 11,22 \pm 0,0 \mathrm{~mm} / \mathrm{d}$ a $30^{\circ} \mathrm{C}$, mientras que HPI- 210 no creció después de $28{ }^{\circ} \mathrm{C}$. Estos resultados sugieren que las tres cepas fueron inducidas a crecer mejor por efecto de los medios suplementados con los insectos; a $28{ }^{\circ} \mathrm{C}$, HPI-210 presentó el mayor crecimiento/d ( $\mathrm{F}=25,24, \mathrm{E} 8, \mathrm{P}=0,0001)$ seguido de $\mathrm{M} 1$ y $\mathrm{B} 1$, mientras que M1 con suplemento de $S$. frugiperda creció mejor a $30^{\circ} \mathrm{C}(\mathrm{F}=38,33, \mathrm{E} 8, \mathrm{P}=0,0001)$. Estos resultados de crecimiento radial, desarrollo macromorfológico y tolerancia a la temperatura permiten seleccionar a las mejores cepas para evaluar su virulencia en insectos plaga.

Palabras clave: Crecimiento radial, entomopatógenos, hongos, Lepidoptera, macromorfología, medio suplementado.

Abstract: The macromorphology of Beauveria bassiana (B1), Metarhizium anisopliae (M1) and Isaria javanica (HPI-210) strains was studied, as well as its radial growth at temperatures of $25,28,30,35$ and $40{ }^{\circ} \mathrm{C}$ in PDA medium supplemented with powder of Diatraea considerata, Spodoptera frugiperda and Galleria mellonella. The fungi were inoculated by adding $2 \mu \mathrm{l}$ of a suspension with $1 \times 10^{7}$ spores $/ \mathrm{ml}$. Afterwards the radial strains growth in $\mathrm{mm} / \mathrm{d}$ was measured during $15 \mathrm{~d}$. To determine the interaction between the three strains, the three lepidopteran powders, control PDA and the five temperatures, a factorial analysis 3 x 4 x 5 was used; fungi growth data was analyzed by ANOVA and Tukey test. The macromorphological characteristics of each species were studied, finding significant statistical differences in the growth at different temperatures, with respect to the control. The optimal growth for the three fungi was at $28^{\circ} \mathrm{C}$. Strain B1 supplemented with $D$. considerata powder grew $2.76 \pm 0.06 \mathrm{~mm} / \mathrm{d}, \mathrm{M} 1, G$. mellonella $2.77 \pm 0.02 \mathrm{~mm} / \mathrm{d}$ and M1 HPI-210 D. considerata $3.27 \pm 0.06 \mathrm{~mm} / \mathrm{d}$. Strains B1 grew $0.45 \pm 0.03 \mathrm{~mm} / \mathrm{d}$ and M1 $1.22 \pm 0.03$ at 30 ${ }^{\circ} \mathrm{C}$, while HPI-210 did not grow after $28{ }^{\circ} \mathrm{C}$. These results suggested that the strains were induced by medium contained insect supplementation; at $28^{\circ} \mathrm{C}$, HPI-210 obtained the best growth/d $(\mathrm{F}=25.24, \mathrm{E} 8, \mathrm{P}=0.0001)$, followed by $\mathrm{M} 1$ and $\mathrm{B} 1$, while M1 supplemented with $S$. frugiperda grew best at $30{ }^{\circ} \mathrm{C}(\mathrm{F}=38.33, \mathrm{E} 8, \mathrm{P}=0.0001)$. These results of radial growth, macromorphology and tolerance of strains at temperature, allow the selection of the best strains to assess its virulence in insect pests.

Keywords: Growth fungi, entomopathogens, fungi, Lepidoptera, macromorphology, medium supplement. 


\section{Introducción}

Los hongos entomopatógenos constituyen un grupo ampliamente diseminado en el medio ambiente que provocan infecciones fungosas a poblaciones de artrópodos (Díaz et al. 2006). Las especies más importantes por su distribución son Beauveria bassiana (Balsamo) Vuillemin y Metarhizium anisopliae (Metschnikoff) Sorokin (Cañedo y Ames 2004). Estos agentes infectan insectos, a través de la penetración de la cutícula, ejerciendo múltiples mecanismos de acción, evitando que el hospedero desarrolle resistencia (MottaDelgado y Murcia-Ordoñez 2011). La producción de hongos para el control de plagas involucra técnicas para su producción masiva, formulación y estrategias de aplicación (Butt y Goettel 2001). Al respecto, Meyling y Eilenberg (2007) afirman que para su utilización como agentes de control biológico es necesario realizar prácticas agrícolas que propicien las condiciones ambientales de humedad relativa y temperatura para favorecer la actividad de infección de los entomopatógenos, además de una selección de cepas con alta patogenicidad y virulencia sobre sus hospederos.

La infección de los hongos depende de las propiedades de la cutícula como el grosor, la esclerotización y la presencia de sustancias antifúngicas y nutricionales (Charnley 1992), así como de otros atributos que determinan su virulencia frente a sus hospedantes (Zibaee y Bandani 2009). También las enzimas específicas que degradan la cutícula como las lipasas, quitinasas, proteasas, quimoelastasas y quimotripsina, tienen relación con la virulencia (Gupta et al. 1994). Respecto a la temperatura, el hongo Isaria javanica (Friederichs \& Bally) Samson \& Hywel-Jones es conocido como hongo mesófilo y algunas especies son más tolerantes a alta temperatura que otras. El crecimiento de Isaria fumosorosea (Wize) es, generalmente, mayor a temperaturas bajas que altas (Fargues et al. 1992). Con relación a esto, varios autores coinciden en que la temperatura óptima de crecimiento de estos hongos se encuentra entre 25 a $30{ }^{\circ} \mathrm{C}$ (Wilding 1970; Vanegas 2006; Bugeme et al. 2008). En este sentido, Ruelas-Ayala et al. (2013) encontraron que entre 28 a $34{ }^{\circ} \mathrm{C}$ se disminuye el crecimiento de $B$. bassiana de 28 a $79 \%$, mientras que en $M$. anisopliae fue de 24 a $31 \%$, por lo tanto este hongo fue más tolerante a temperaturas altas. Por lo anterior, es recomendable combinar dos o más cepas de hongos como agentes de control en campo, en función a sus adaptaciones a su entorno para que los aislamientos funcionen (Seid et al. 2019).

Algunas especies de hongos pueden confundirse con otros géneros, dependiendo de su condición reproductiva sexual o asexual; al respecto, en $M$. anisopliae, estudios filogenéticos junto con evidencias morfológicas soportan la monofilia de nuevos taxa. Kepler et al. (2014) realizaron estudios moleculares para establecer la relación de este género con otras especies. También Bischoff et al. (2009) evaluaron la filogenia del complejo $M$. anisopliae e identificaron nueve especies mediante la morfología y secuencias de los genes EF-1, RPB1, RPB2.

Respecto al crecimiento de hongos en medios suplementados, González García et al. (2001) observaron aumentos significativos de patogenicidad de cuatro aislados de B. bassiana, cuando se subcultivaron en medio suplementado con integumento de broca del café Hipothenemus hampei (Ferrari, 1867), variando de 80 a 100\% con un tiempo letal de 3,2 y 3,7 días, respectivamente; confirmando que el integumento actúa como fuente de nutrición de algunos hongos aumentando su patogenicidad. Por otro lado, Vejar-Cota et al. (2017) caracterizaron morfológicamente cepas de B. bassiana, $M$. anisopliae e $I$. javanica, además midieron durante 21 días su crecimiento, encontrando que el intervalo de crecimiento de estas cepas varió entre 2,05 y 3,03 mm/día. Torres de la C. et al. (2013) encontraron una tasa de crecimiento de 2,63 \pm $0,18 \mathrm{~mm} /$ día a $25^{\circ} \mathrm{C}$ para 17 aislamientos de $M$. anisopliae.

Por otro lado, se ha estudiado la relación de la virulencia de cepas de $B$. bassiana y $M$. anisopliae con respecto a la tolerancia a temperatura (Fargues et al. 1992; McCammon y Rath 1994; Selman et al. 1997; Vidal et al. 1997). Seid et al. (2019) al evaluar la germinación y tasa de crecimiento de seis cepas de $B$. bassiana de origen ártico y tropical a 8 y $12{ }^{\circ} \mathrm{C}$, encontraron que los aislamientos de origen ártico mostraron mayor germinación y tasa de crecimiento que las de los trópicos. Tres de los aislamientos se probaron in vivo para determinar la temperatura de infección sobre larvas de Tenebrio molitor L., 1758 (Coleoptera: Tenebrionidae), los resultados indicaron que las condiciones en el medio natural no reflejan directamente el efecto del ambiente biótico como la infección del huésped, por lo que la selección de aislados se debe basar en la virulencia y también en consideraciones de la condición abiótica para que los aislamientos funcionen. Por lo anterior, el objetivo del presente trabajo fue inducir el desarrollo de cepas de B. bassiana, M. anisopliae e I. javanica con medios de cultivo suplementados con polvos de tres insectos plaga, y evaluar su macromorfología, crecimiento radial y tolerancia a la temperatura.

\section{Materiales y métodos}

Selección de cepas y viabilidad de esporas. Las cepas B1 (B. bassiana), M1 (M. anisopliae) y HPI-210 (I. javanica) fueron obtenidas de la colección de hongos entomopatógenos del laboratorio de Bioinsecticidas del CIIDIR-IPN Unidad Sinaloa. La viabilidad de las esporas se determinó de acuerdo con el método de Inglis et al. (2012), la solución de esporas se puso en agitación en un tubo eppendorf de 1,5 $\mathrm{ml}$ con agua destilada, a partir de esta se hicieron soluciones seriadas para obtener 1 x $10^{7}$ conidios $/ \mathrm{ml}$. La suspensión se sembró en cajas Petri con una capa delgada de PDA $(10 \mathrm{~mm})$ dentro de una campana de bioseguridad (Telstar bio-11-A). Las cajas se incubaron a $28{ }^{\circ} \mathrm{C}$ en una cámara bioclimática (Felisa $\left.{ }^{\circledR}\right)$ por un periodo de 18-24 h. Después se cortó $1 \mathrm{~cm}^{2}$ de PDA con el hongo y se colocó sobre un portaobjetos para observar la muestra en un microscopio óptico (BOECO) a 40X y contar el número de conidios germinados. Se registraron solo los conidios cuyo tubo germinativo fue dos o más veces mayor al diámetro del conidio, se consideró una cepa viable a aquella con un número de conidios germinados mayor a $90 \%$ (Vélez et al. 1997).

Preparación del suplemento. Se utilizaron larvas y pupas del barrenador de la caña de azúcar Diatrea considerata (Heinrich, 1931), del gusano cogollero Spodoptera frugiperda (J. E. Smith, 1797) y del gusano de la cera Galleria mellonella (L., 1756), provenientes del laboratorio de Bioinsecticidas del CIIDIR-IPN Unidad Sinaloa. Los insectos se sacrificaron por enfriamiento y después de secaron en una estufa (JSGI-10T) a $50{ }^{\circ} \mathrm{C}$ durante tres días, al retirarlos se molieron en un mortero hasta obtener un polvo fino que se pasó a través de una malla de $500 \mathrm{~mm}$. El polvo así obtenido fue secado en una estufa a $50{ }^{\circ} \mathrm{C}$ durante $24 \mathrm{~h}$ para eliminar la humedad restante. 
Elaboración del medio de cultivo suplementado. A un vaso de precipitados con $500 \mathrm{ml}$ de agua destilada se le agregaron 19,5 g de agar dextrosa papa (PDA) y se adicionaron $2 \mathrm{~g}$ de suplemento del polvo del insecto correspondiente. El caldo preparado se colocó en una plancha de calentamiento con agitación (CIMAREC), posteriormente se esterilizó en un autoclave (All American $25-\mathrm{x}$ ) por $15 \mathrm{~min}$ a $120{ }^{\circ} \mathrm{C}$ y una presión de $1,25 \mathrm{Kg} / \mathrm{cm}^{2}$. El medio de cultivo se vació en cajas Petri estériles, vertiendo $20 \mathrm{ml}$ de cada uno de ellos bajo una cámara de flujo laminar (Cobeal HLV no. 04010-INP-3,5AMP).

Cosecha y concentración de conidios. Los conidios de las cepas B1, M1 y HPI-210 de las cajas Petri fueron cosechados y puestos en tubos Falcón con $10 \mathrm{ml}$ de Tween 80 al 0,25\%, en una campana de bioseguridad (Telstar bio-11-A). Se hicieron diluciones hasta obtener $1 \times 10^{7}$ esporas $/ \mathrm{ml}$; se colocaron 300 $\mu l$ de la suspensión de conidios a tubos eppendorf, la cual se usó como suspensión madre para hacer diluciones seriadas, de cada una de estas se tomaron $15 \mu \mathrm{l}$ y se pusieron entre el cubreobjetos y la cámara de Neubauer ( 30 x 70 y $4 \mathrm{~mm})$. El conteo de conidios se realizó en un microscopio óptico marca BOECO a 40 y $100 \mathrm{X}$.

Macromorfología de las cepas. Las características morfológicas de color y aspecto de las cepas de B. bassiana $y$ $M$. anisopliae suplementadas se hizo mediante la observación microscópica, una vez que se alcanzó el máximo desarrollo de los hongos, se comparó su morfología con las características descritas por Alcocer (1979), Brady (1979) y Padilla et al. (2000). En el caso de la cepa de I. javanicus se usaron las claves de Brown y Smith (1957) y Shimazu y Takatsuka (2010).

Siembra y desarrollo de las cepas. Para calcular el crecimiento radial se consideraron aspectos utilizados por
Zervakis et al. (2001) y Ruelas-Ayala et al. (2013). En términos generales el procedimiento fue el siguiente: en el punto central de las cajas Petri, para cada cepa se colocaron discos de papel filtro estéril de $6 \mathrm{~mm}$ de diámetro, en cada disco se pusieron $2 \mu \mathrm{l}$ de la suspensión conidial, las cajas inoculadas se incubaron en una cámara bioclimática (Telstar bio-11-A), la lectura del crecimiento de las cepas en las cajas se realizó cada 24 h durante 15 días; la variable de crecimiento en $\mathrm{mm} / \mathrm{d}$ se hizo midiendo el diámetro de la colonia en dos ejes cardinales marcados al reverso de las cajas con un vernier Petrul ${ }^{\circledR}($ VER-6PX).

Evaluación del desarrollo de las cepas a diferentes temperaturas. Se evaluó el crecimiento radial de las diferentes cepas en medio PDA con suplemento de cada insecto y un control PDA. En cada caja se colocó un círculo de $6 \mathrm{~mm}$ de diámetro con $2 \mu \mathrm{l}$ de la suspensión de conidios, a $25,28,30,35$ y $40{ }^{\circ} \mathrm{C}$, estas se incubaron en la cámara bioclimática. La lectura del crecimiento de las cepas en las cajas se realizó cada $24 \mathrm{~h}$ durante 15 días; la variable de crecimiento en $\mathrm{mm} / \mathrm{d}$ se hizo midiendo el diámetro de la colonia en dos ejes cardinales marcados al reverso de las cajas con el mismo vernier (Ruelas-Ayala et al. 2013).

Análisis estadísticos. Para comparar los datos de crecimiento radial de las diferentes cepas en los medios con suplemento, se utilizó un diseño estadístico de bloques completamente al azar. Para determinar la interacción entre las tres cepas, las tres especies de insectos más el control y las cinco temperaturas se utilizó un análisis factorial $3 \times 4 \times 5$ con tres repeticiones por cepa, el análisis de los datos se hizo mediante un ANOVA y para determinar diferencias significativas entre las medias se aplicó la prueba de rango múltiple de Tukey $(\alpha=0,05)$ usando el programa SAS v.9 (SAS Institute Inc. 2009).

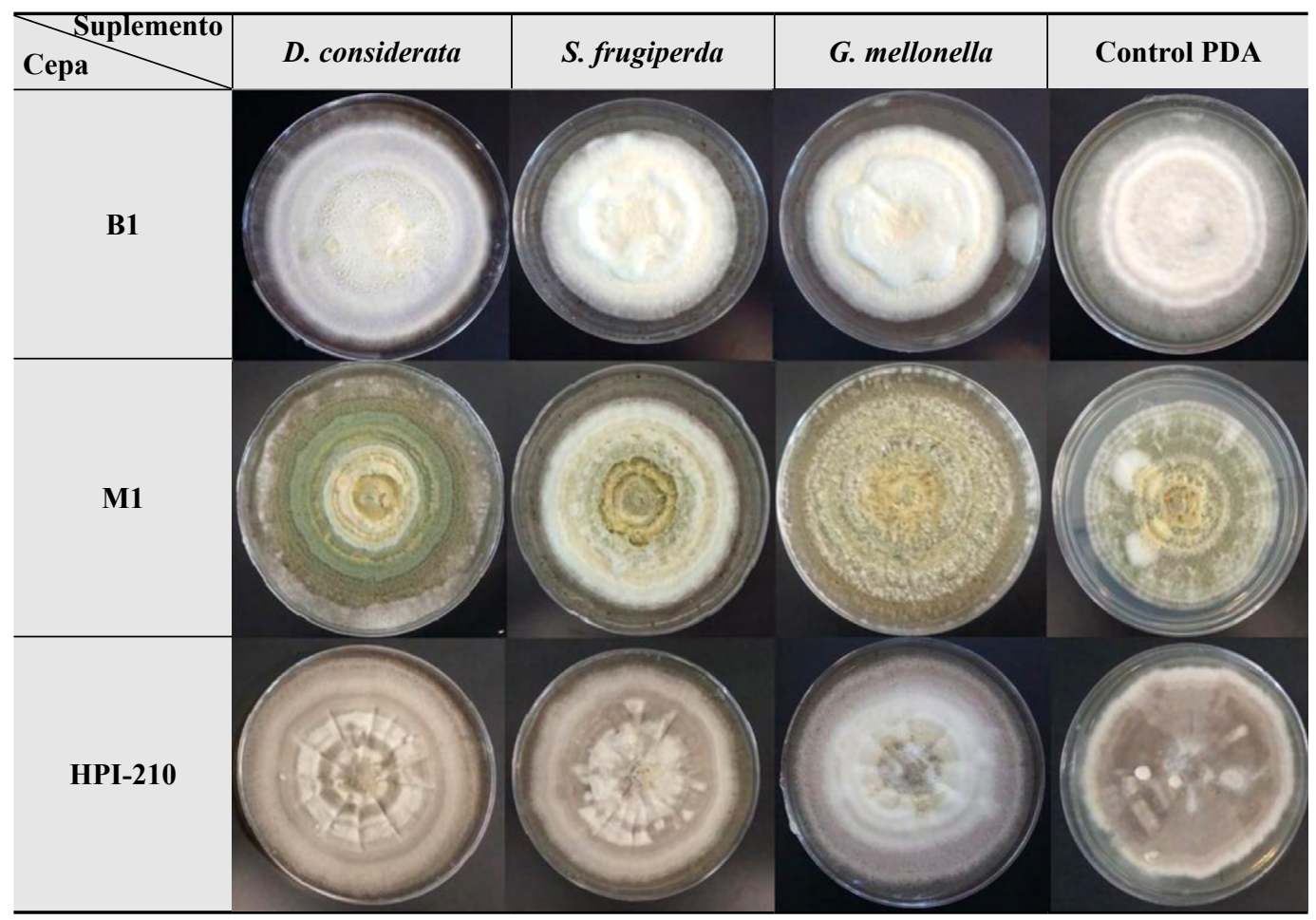

Figura 1. Crecimiento radial de B1 (Beauveria bassiana), M1 (Metarhizium anisopliae), HPI-210 (Isaria javanica) en PDA suplementado con polvo de Diatraea considerata, Spodoptera frugiperda, Galleria mellonella y PDA-control a los 15 días de desarrollo de la colonia. 


\section{Resultados y discusión}

Macromorfología. Para asegurar que se trabajó con las cepas adecuadas, se corroboró que las características morfológicas correspondieran a las de las claves taxonómicas mencionadas para cada especie (Fig. 1) observando el aspecto de las colonias de las cepas de B. bassiana (B1), M. anisopliae (M1) y I. javanica (HPI-210). La textura que presentaron las colonias de B1 al inicio de su desarrollo fue algodonosa de color blanco a beige, cambiando a amarillas pulverulentas durante el crecimiento micelial. Las características de la cepa B1 coincidieron con las descritas por Alcocer (1979). En el caso de M1 la morfología se mantuvo en una textura algodonosa formando halos de color blanco, beige y verde en la maduración de la cepa. La cepa HPI-210 inicialmente fue de aspecto algodonoso blanco, tornándose gris después de esporular. Respecto a M1 Brady (1979) menciona que M. anisopliae en PDA presenta micelio con borde blanco, mientras que Padilla et al. (2000) obtuvieron colonias algodonosas, aterciopeladas y compactas; en el caso de la cepa HPI-210 I. javanicus se observó que sus características corresponden a las descritas por Brown y Smith (1957) y Shimazu y Takatsuka (2010).

Efecto del suplemento y la temperatura. En la Tabla 1 se muestran los resultados estadísticos del crecimiento de las cepas con los suplementos a diferentes temperaturas. Los mejores resultados del efecto del suplemento y la temperatura en el crecimiento de las cepas se obtuvo a $28^{\circ} \mathrm{C}$; en el caso de B1 al utilizar el suplemento de $D$. considerata se obtuvo un crecimiento de $2,76 \pm 0,06 \mathrm{~mm} / \mathrm{d}$ y con HPI-210 3,27 \pm $0,06 \mathrm{~mm} / \mathrm{d}$, mientras que para M1 $(2,62 \pm 0,08 \pm \mathrm{mm} / \mathrm{d})$. A $30{ }^{\circ} \mathrm{C}$ B1 creció $0,45 \pm 0.03 \mathrm{~mm} / \mathrm{d}$ en el mismo medio suplementado y con M1 1,22 $\pm 0,03 \mathrm{~mm} / \mathrm{d}$ con $S$. frugiperda. No se observó crecimiento de ninguna de las cepas a $35 \mathrm{y}$ $40{ }^{\circ} \mathrm{C}$. El crecimiento diario de estas cepas fue superior al obtenido por Vejar-Cota et al. (2017) quienes determinaron un crecimiento sin suplementos de B. bassiana de $2,29 \mathrm{~mm}$ y para I. javanica (HPI-210) 2,07 mm/d. Por otro lado, Torres de la C. et al. (2013) encontraron una tasa de crecimiento micelial de $M$. anisopliae similar a nuestros resultados.

Estos resultados indicaron que las tres cepas fueron inducidas para crecer mejor en los medios suplementados con los tres insectos, este efecto se observó en la morfología de las cepas de los tres hongos (Fig. 1). En este sentido, González García et al. (2001) observaron incrementos significativos en el crecimiento de cuatro aislados de $B$. bassiana cuando se cultivaron en medio suplementado con integumento de broca del café, donde este aumento varió de 80 a $100 \%$ con un tiempo letal de 3,2 y 3,7 días, confirmando que el integumento actúa como fuente de nutrición de algunos hongos.

En relación al efecto de la temperatura en el crecimiento de los hongos, varios autores coinciden en que la temperatura óptima es de 25 a $30{ }^{\circ} \mathrm{C}$ (Wilding 1970; Vanegas 2006; Bugeme et al. 2008). I. javanica con D. considerata mostró mayor crecimiento a $28{ }^{\circ} \mathrm{C} 3,27 \pm 0,06$ y no presentó crecimiento a partir de $\operatorname{los} 30^{\circ} \mathrm{C}$. No obstante, I. fumosorosea crece mejor a temperaturas bajas que altas (Fargues et al. 1992). En todos los casos se observó bajo crecimiento a 30 ${ }^{\circ} \mathrm{C}$ y no hubo crecimiento a 35 y $40{ }^{\circ} \mathrm{C}$. Al respecto, RuelasAyala et al. (2013) encontraron que la tasa de disminución del crecimiento de $B$. bassiana fue de 28 a $79 \%$ y en $M$. anisopliae de 24 a $31 \%$, siendo esta última más tolerante a altas temperaturas.

En el estudio para determinar el efecto de los suplementos a diferentes temperaturas, el ANOVA indicó que la cepa M1 con suplemento de $S$. frugiperda fue la más tolerante a temperatura de $30{ }^{\circ} \mathrm{C}(\mathrm{F}=38,33, \mathrm{E} 8, \mathrm{P}=0,0001)$ (Tabla 2), por lo que los hongos B1 y M1 mostraron tolerancia a esta temperatura; esto se puede deber a que son originarios de Sinaloa, donde la temperatura media anual es de $25{ }^{\circ} \mathrm{C}$ y la máxima varía de 44 a $47{ }^{\circ} \mathrm{C}$; mientras que HPI-210 es proveniente del estado de Morelos, donde se registran temperaturas de 21 a $32{ }^{\circ} \mathrm{C}$. Con base a esto, se puede suponer que existe una relación estrecha entre lugares de origen y la tolerancia a altas temperaturas, como lo sugieren otros estudios (Fargues et al. 1992; McCammon y Rath 1994; Selman et al. 1997; Vidal et al. 1997; Ruelas-Ayala et al. 2013).

Tabla 1. Crecimiento radial de Beauveria bassiana, Metarhizium anisopliae e Isaria javanica suplementado con polvo de macerado de tres insectos a diferente temperatura.

\begin{tabular}{|c|c|c|c|c|}
\hline \multirow{2}{*}{ Cepa } & \multirow{2}{*}{ Tratamiento polvo } & \multicolumn{3}{|c|}{ Crecimiento radial $(\bar{X} \pm E E)^{1,2,3}$} \\
\hline & & $25^{\circ} \mathrm{C}$ & $28^{\circ} \mathrm{C}$ & $30{ }^{\circ} \mathrm{C}$ \\
\hline \multirow{4}{*}{ B1 } & $\mathrm{PDA}+$ D. considerata & $2,59 \pm 0,02 \mathrm{ab}$ & $2,76 \pm 0,06 \mathrm{a}$ & $0,45 \pm 0,03 \mathrm{f}$ \\
\hline & $\mathrm{PDA}+S$. frugiperda & $2,63 \pm 0,02 \mathrm{ab}$ & $2,63 \pm 0,11 \mathrm{ab}$ & $0,40 \pm 0,04 \mathrm{f}$ \\
\hline & $\mathrm{PDA}+$ G. mellonella & $2,20 \pm 0,02 \mathrm{~d}$ & $2,44 \pm 0,02 \mathrm{bc}$ & $0,14 \pm 0,07 \mathrm{~g}$ \\
\hline & PDA & $2,29 \pm 0,03 \mathrm{~cd}$ & $1,80 \pm 0,03 \mathrm{e}$ & $0,40 \pm 0,07 \mathrm{f}$ \\
\hline \multirow{4}{*}{ M1 } & $\mathrm{PDA}+$ D. considerata & $2,64 \pm 0,06 \mathrm{a}$ & $2,62 \pm 0,08 \mathrm{ab}$ & $0,86 \pm 0,07 \mathrm{de}$ \\
\hline & $\mathrm{PDA}+$ S. frugiperda & $2,41 \pm 0,37 \mathrm{ab}$ & $2,46 \pm 0,04 a b$ & $1,22 \pm 0,03 \mathrm{~d}$ \\
\hline & $\mathrm{PDA}+$ G. mellonella & $2,72 \pm 0,06 \mathrm{a}$ & $2,77 \pm 0,02 \mathrm{a}$ & $0,48 \pm 0,03 \mathrm{e}$ \\
\hline & PDA & $2,24 \pm 0,11 \mathrm{~b}$ & $1,80 \pm 0,07 \mathrm{c}$ & $0,87 \pm 0,04 \mathrm{~d}$ \\
\hline \multirow{4}{*}{ HPI-210 } & $\mathrm{PDA}+D$. considerata & $3,07 \pm 0,07 \mathrm{ab}$ & $3,27 \pm 0,06 \mathrm{a}$ & $*$ \\
\hline & $\mathrm{PDA}+S$. frugiperda & $3,03 \pm 0,17 \mathrm{abc}$ & $2,81 \pm 0,10 \mathrm{bc}$ & $*$ \\
\hline & $\mathrm{PDA}+$ G. mellonella & $2,76 \pm 0,05 \mathrm{c}$ & $3,02 \pm 0,03 \mathrm{abc}$ & $*$ \\
\hline & PDA & $2,92 \pm 0,13 \mathrm{bc}$ & $1,94 \pm 0,12 \mathrm{~d}$ & $*$ \\
\hline
\end{tabular}


Tabla 2. Valores de F y (P) del ANOVA realizado para determinar el efecto de los suplementos en las diferentes cepas a tres temperaturas.

\begin{tabular}{lccc}
\hline \multirow{2}{*}{ Cepa } & \multicolumn{3}{c}{ Temperaturas } \\
\cline { 2 - 4 } & $\mathbf{2 5}^{\circ} \mathbf{C}$ & $\mathbf{2 8}^{\circ} \mathbf{C}$ & $\mathbf{3 0}^{\circ} \mathbf{C}$ \\
\hline Beauveria bassiana B1 & $18,73(0,0006)$ & $48,76(0,0001)$ & $5,43(0,2449)$ \\
Metarhizium anisopliae M1 & $3,10(0,0899)$ & $20,7(0,0004)$ & $38,33(0,0001)$ \\
Isaria javanica HPI-210 & $2,75(0,1120)$ & $25,24(0,0001)$ & $41,0(0,0001)$ \\
\hline
\end{tabular}

\section{Conclusiones}

El uso de medios suplementados con polvo de las tres especies de lepidópteros logró inducir el desarrollo óptimo de cepas de B. bassiana (B1), M. anisopliae (M1) e I. javanica (HPI-210) a $28{ }^{\circ} \mathrm{C}$. Las cepas B1 y M1 crecieron más a $30{ }^{\circ} \mathrm{C}$ en los tratamientos con suplementos de D. considerata y $S$. frugiperda, en HPI-210 no hubo crecimiento. Las cepas B1 y HPI-210 con D. considerata mostraron el mayor crecimiento radial; M1 creció más con $G$. mellonella a $28{ }^{\circ} \mathrm{C}$. El medio suplementado con polvo de estos insectos logró también aumentar la tasa de crecimiento de los hongos a las tres temperaturas, lo que permite seleccionar a alguna de estas cepas con base en su macromorfología, mayor crecimiento radial y tolerancia a la temperatura para evaluar su virulencia contra insectos plaga.

\section{Agradecimientos}

Se agradece a la SIP - IPN, el apoyo financiero otorgado para la realización de este trabajo, a través del proyecto: "Estudio del modo de acción de formulaciones de hongos y nematodos entomopatógenos en insectos plaga de importancia agrícola en Sinaloa", clave SIP: 20160476. A M. en C. Alicia Chávez Medina por la colaboración en actividades del formato del artículo.

\section{Literatura citada}

ALCOCER, G. L. 1979. Incidencia del hongo Beauveria bassiana en México y su producción en laboratorio para el control microbiano inducido. DGSV-SARH. pp. 107-110. VII Reunión Nacional de Control Biológico. Veracruz, México.

BISCHOFF, J. F.; REHNER, S. A.; HUMBER, R. A. 2009. A multilocus phylogeny of the Metarhizium anisopliae lineage. Mycologia 101 (4): 512-530. https://doi.org/10.3852/07-202

BRADY, B. L. K. 1979. Metarhizium anisopliae. Commonwealth Mycological Institute, Descriptions of Pathogenic Fungi and Bacteria. $\mathrm{N}^{\circ}$ 609: 2 p.

BROWN, A. H. S.; SMITH, G. 1957. The genus Paecilomyces Bainier and its perfect stage Byssochlamys Westing. Transactions of British Mycological Society 40 (1): 17-89. https://doi. org/10.1016/S0007-1536(57)80066-7

BUGEME, D. M.; MANIANIA, N. K.; KNAPP, M.; BOGA, H. I. 2008. Effect of temperature on virulence of Beauveria bassiana and Metarhizium anisopliae isolates to Tetranychus evansi. Experimental and Applied Acarology 46: 275-285. https://doi. org/10.1007/s10493-008-9179-1

BUTT, T. M.; GOETTEL, M. S. 2001. Bioassays of entomogenous fungi. pp. 141-196. En: Navon, A.; Ascher, K. R. (Eds.). Bioassays of entomopathogenic microbes and nematodes. CAB International, Wallingford, Reino Unido. 315 p. https://doi. org/10.1079/9780851994222.0141

CAÑEDO, V.; AMES, T. 2004. Medios de cultivos de laboratorio: Manual de laboratorio para el manejo de hongos entomopatógenos. Perú: Centro Internacional de la Papa (CIP), $18-22$.

CHARNLEY, A. K. 1992. Mechanism of fungal pathogenesis in insects with particular reference to locusts. pp. 191-199. En: Lomer, C. J.; Prior, C. (Eds.). Biological control of locusts and grasshoppers. Melkshan, Reino Unido: CAB International 399 p.

DÍAZ, M. P.; FLORES, M.; RODRÍGUEZ, N. S.; DE LA TORRE, M. 2006. Mecanismo de acción de los hongos entomopatógenos. INCI 31 (12): 856-860.

FARGUES, J.; MANIANIA, N. K.; DELMAS, J. C.; SMITS, N. 1992. Influence de la température sur la croissance in vitro d'hyphomycètes entomopathogènes. Agronomie 12 (7): 557564. https://doi.org/10.1051/agro:19920708

GONZÁLEZ GARCÍA, M. T.; VALENCIA JIMÉNEZ, A.; BUSTILLO PARDEY, A. E. 2001. Incremento de la patogenicidad de Beauveria bassiana sobre Hypothenemus hampei, utilizando integumento del insecto en el medio de cultivo. Manejo Integrado de Plagas (Costa Rica) 60: 31-35.

GUPTA, S. C.; LEATHERS, T. D.; SAYED, G. N.; IGNOFFO, C. M. 1994. Relationship among enzyme activities and virulence parameters in Beauveria bassiana Infections of Galleria mellonella and Trichoplusia ni. Journal of Invertebrate Pathology 64 (1): 13-17. https://doi.org/10.1006/jipa.1994.1062

INGLIS, G. D.; ENKERLI, J.; GOETTEL, M. S. 2012. Laboratory techniques used for entomopathogenic fungi: Hypocreales. pp. 189-253. En: Lacey, L. A. (Ed.). Manual of techniques in invertebrate pathology. Second edition, Chapter VII. Academic Press, Londres 504 p. https://doi.org/10.1016/B978-0-12386899-2.00007-5

KEPLER, R. M.; HUMBER, R. A.; BISCHOFF, J. F.; REHNER, S. A. 2014. Clarification of generic and species boundaries for Metarhizium and related fungi through multigene phylogenetics. Mycologia 106 (4): 811-829. https://doi.org/10.3852/13-319

McCAMMON, S. A.; RATH, A. C. 1994. Separation of Metarhizium anisopliae strains by temperature dependent germination rates. Mycological Research 98 (11): 1253-1257. https://doi. org/10.1016/S0953-7562(09)80295-5

MEYLING, N. V.; EILENBERG, J. 2007. Ecology of the entomopathogenic fungi Beauveria bassiana and Metarhizium anisopliae in temperate agroecosystems: potential for conservation biological control. Biological Control 43 (2): 145155. https://doi.org/10.1016/j.biocontrol.2007.07.007

MOTTA-DELGADO, P. A.; MURCIA-ORDOÑEZ, B. 2011. Hongos entomopatógenos como alternativa para el control biológico de plagas. Revista Ambiente \& Água 6 (2): 77-90. https://doi.org/10.4136/ambi-agua.187

PADILLA M., G. N.; BERNAL U., M. G.; VÉLEZ A., P. E.; MONTOYA R., E. C. 2000. Caracterización patogénica y morfológica de aislamientos de Metarhizium anisopliae obtenidos de diferentes órdenes insectiles. Cenicafé 51 (1): 2840.

RUELAS-AYALA， R. D.; GARCÍA-GUTIÉRREZ, C.; ARCHULETA-TORRES, A. 2013. Selection of Beauveria bassiana and Metarhizium anisopliae isolates tolerant to high temperatures for the control of Sitophilus zeamais (Coleoptera: Curculionidae). Southwestern Entomologist 38 (2): 313-324. https://doi.org/10.3958/059.038.0214

SAS Institute Inc. 2009. SAS/STAT ® 9.1 User's Guide. Cary, NC: SAS Institute Inc.

SEID, A. M.; FREDENSBORG, B. L.; STEINWENDER, B. M.; MEYLING, N. V. 2019. Temperature-dependent germination, growth and co-infection of Beauveria spp. isolates from different climatic regions. Biocontrol Science and Technology 29 (5): 411-426.

SELMAN, B. J.; DAYER, M. S.; HASAN, M. 1997. Pathogenicity of Beauveria bassiana (Bals.) Vuill. to the larvae of diamondback moth, Plutella xylostella L. (Lep., Yponomeutidae). Journal of Applied Entomologist 121 (1-5): 47-49. https://doi. org/10.1111/j.1439-0418.1997.tb01369.x 
SHIMAZU, M.; TAKATSUKA, J. 2010. Isaria javanica (anamorphic Cordycipitaceae) isolated from gypsy moth larvae, Lymantria dispar (Lepidoptera: Lymantriidae), in Japan. Applied Entomology and Zoology 45 (3): 497-504. https://doi. org/10.1303/aez.2010.497

TORRES DE LA C., M.; CORTEZ, M. H.; ORTÍZ, G. C. F.; CAPPELLO, G. S.; DE LA CRUZ, P. A. 2013. Caracterización de aislamientos nativos de Metarhizium anisopliae y su patogenicidad hacia Aeneolamia postica en Tabasco, México. Revista Colombiana de Entomología 39 (1): 40-46. https://doi. org/10.25100/socolen.v39i1.8074

VANEGAS, A. J. A. 2006. Evaluación de hongos entomopatógenos como controladores biológicos de Scutigerella inmaculata. Tesis para obtener el título de Microbiólogo Agrícola y Veterinaria. Pontificia Universidad Javeriana, Facultad de Ciencias, Bogotá D. C., 79 p.

VEJAR-COTA, G.; GARCÍA-GUTIÉRREZ, C.; ROSAS-GARCÍA, N. M.; ESCOBEDO-BONILLA, C. M.; GONZÁLEZOCAMPO, H. A. 2017. Morphological and molecular characterization of entomopathogenic fungi with potential to control sugarcane borers at Sinaloa. Southwestern Entomologist 42 (2): 395-400. https://doi.org/10.3958/059.042.0208

VÉLEZ A., P. E.; POSADA F., J. F.; MARÍN M., P.; GONZÁLEZ G., M. T.; OSORIO V., E.; BUSTILlO P., A. E. 1997. Técnicas para el control de calidad de formulaciones de hongos entomopatógenos. Centro Nacional de Investigaciones del café. Boletín Técnico 17: 37.

VIDAL, C.; FARGUES, J.; LACEY, L. A. 1997. Intraspecific variability of Paecilomyces fumosoroseus: effect of temperature on vegetative growth. Journal of Invertebrate Pathology 70 (1): 18-26. https://doi.org/10.1006/jipa.1997.4658

WILDING, N. 1970. Entomophtora conidia in the air- spora. Journal of General Microbiology 62: 149-153. https://doi. org/10.1099/00221287-62-2-149

ZERVAKIS, G.; PHILIPPOUSSIS, A.; IOANNIDOU, S.; DIAMANTOPOULOU, P. 2001. Mycelium growth kinetics and optimal temperature conditions for the cultivation of edible mushroom species on lignocellulosic substrates. Folia Microbiologica 46: 231. https://doi.org/10.1007/BF02818539

ZIBAEE, A.; BANDANI, A. R. 2009. Purification and characterization of the cuticle-degrading protease produced by the entomopathogenic fungus, Beauveria bassiana in the presence of sunn pest, Eurygaster integriceps (Hemiptera: Scutelleridae) cuticle. Biocontrol Science and Technology 19 (8): 797-808. https://doi.org/10.1080/09583150903132172

\section{Origen y financiación}

El presente trabajo derivó de la línea de investigación con hongos entomopatógenos que se desarrolla en el laboratorio de bioinsecticidas del CIIDIR Sinaloa, en el que se realizó la tesis de licenciatura de Marina Isabel García Guajardo, estudiante de la UDO, bajo la Dirección del Dr. Cipriano García Gutiérrez y Dr. Guadalupe Vejar Cota como Directores externos y del Dr. Lorenzo Meza García como Director interno. El apoyo financiero fue de la SIP - IPN, a través del proyecto: "Estudio del modo de acción de formulaciones de hongos y nematodos entomopatógenos en insectos plaga de importancia agrícola en Sinaloa”, clave SIP: 20160476.

\section{Contribución de los autores}

Cipriano García-Gutiérrez: Dirección, diseño y supervisión del trabajo experimental, revisión de resultados y redacción del artículo.

Marina Isabel García-Guajardo: Realización del trabajo experimental y procesamiento de datos e imágenes, revisión del artículo.

Guadalupe Vejar-Cota: Dirección, diseño y supervisión del trabajo experimental, procesamiento estadístico de datos e imágenes, revisión del artículo.

Lorenzo Meza-García: Revisión y sugerencias al artículo.

Jesús Alicia Chávez-Medina: Búsqueda de bibliografía, apoyo en el formato del escrito y revisión del artículo. 